\title{
Physicochemical studies of E1(53-66) synthetic peptide by phospholipid monolayers and differential scanning calorimetry
}

\author{
S. Perez ${ }^{2}$, C. Mestres ${ }^{1}$, M. Pujol ${ }^{1}$, M.A. Alsina ${ }^{1}$ and I. Haro ${ }^{2}$ \\ ${ }^{1}$ Faculty of Pharmacy, University of Barcelona, Av. Joan XXIII, s/n, \\ 08028 Barcelona, Spain \\ ${ }^{2}$ Department of Peptide \& Protein Chemistry, Institute for Chemical and \\ Environmental Research (IIQAB-CSIC), Jordi Girona 18-26, 08034 Barcelona, Spain
}

\begin{abstract}
In the present work, the physicochemical characterization of a hepatitis G virus (HGV) synthetic peptide by means of two different techniques was studied: lipid monolayers and differential scanning calorimetry (DSC). We have studied the surface activity of E1(53-66), and its palmitoylated derivative, PalmE1(53-66), and the interactions of the last one with dipalmiltoyl phosphatidylcholine (DPPC), and dipalmitoyl phosphatidylglycerol (DPPG) monolayers. Moreover, DSC was used to study the interaction of PalmE1(53-66) with multilamellar liposomes composed of DPPC.
\end{abstract}

\section{INTRODUCTION}

Hepatitis $\mathrm{G}$ virus (HGV) is a positive-strand RNA virus belonging to the Flaviviridae family. It codifies structural (E1 and E2), and nonstructural (NS2, NS3, NS4, NS5) proteins. According to Hoop\&Woods [1] and the parameters of Welling et al., the sequence E1(53-66) showed a good antigenicity profile and the presence of beta turns [2].

We describe and discuss the physicochemical characterization of E1(53-66) synthetic peptide and its palmitoyled derivative PalmE1(53-66), belonging to the HGV estructural 1 (E1) protein. The aim of this paper is to obtain an insight into the interaction of the peptide with biomembranes.

\section{MATERIALS AND METHODS}

\subsection{Peptide synthesis}

E1(53-66) peptide was synthesized manually on a Rink amide $(0.65 \mathrm{meq} / \mathrm{g})$ resin by solidphase methodology following an Fmoc/tBut strategy by means of a N,N'diisopropylcarbodiimide/1-hidroxybenzotriazole (DIPCDI/HOBt) activation.

The palmitoyl derivative was also obtained in solid phase by using palmitic acid and DIPCDI/HOBt coupling reagents in dimethylformamide/dichloromethane, as previously described [3]. The peptides were synthesized as peptide amides, its purity controlled by amino acid analysis, mass spectrometry and HPLC [4].

\subsection{Physichochemical studies}

Surface pressure experiments were made by the Wilhelmy platinum plate method using a Langmuir balance (KSV 5000), as it was described [5]. Lipid monolayers were spread from methanol/chloroform solutions on a Teflon trough containing PBS (phosphate buffered saline) pH 7.4 or PBS pH 7.4 with PalmE1(53-66). The desired amount of lipid was injected with a Hamilton syringe at the air/water interface. The organic solvent was allowed to evaporate for $15 \mathrm{~min}$ before the film was compressed at a constant compression rate. After each experiment the trough was thoroughly cleaned with ethanol and distilled water. All measurements were 
carried out at room temperature. DSC studies were performed on a Mettler Toledo DSC 821E instrument.

\section{RESULTS AND DISCUSSION}

\subsection{Surface activity}

E1(53-66) did not present surface activity at concentrations from 0.267 to $2.660 \mu \mathrm{M}$. These results were in agreement with the high solubility of peptide in aqueous solutions. Moreover, lipophilic derivative, PalmE1(53-66) presented surface activity at concentrations from 0.045 to $0.906 \mu \mathrm{M}$, with a maximum around $30 \mathrm{mN} / \mathrm{m}$.

\subsection{Compression isotherms}

In DPPC monolayers (Fig. 1) the peptide produced an increase of area/molecule for pressure values below $40 \mathrm{mN} / \mathrm{m}$. At higher pressures the area is lower than pure DPPC, suggesting that the peptide and perhaps some DPPC molecules were ejected to the subphase [6].

For DPPG monolayers (Fig.2), the increase of area/molecule values was observed through all the compression process. These results indicated that the peptide remains in the monolayer.

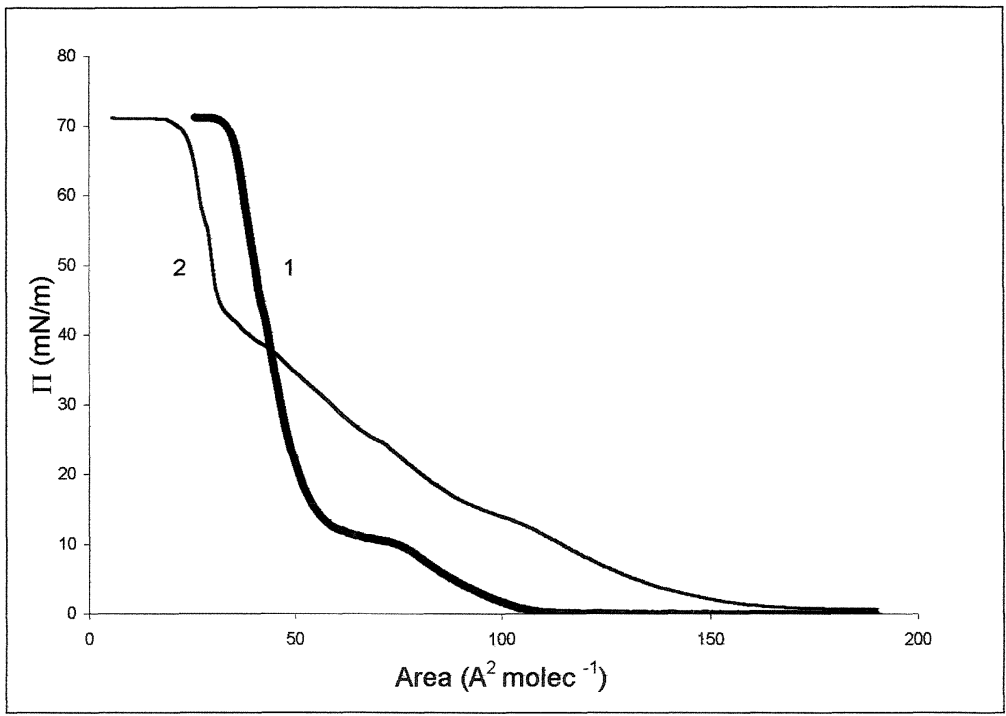

Figure 1. Compression isotherm of 1)DPPC subphase PBS 2)DPPC subphase PalmE1(53-66) 


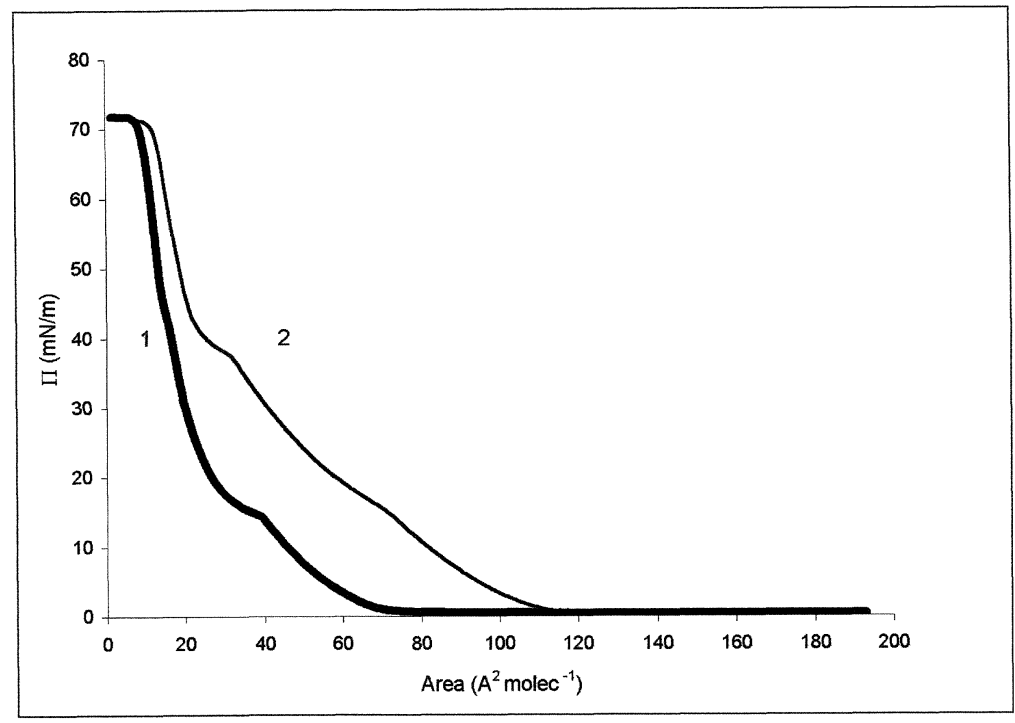

Figure 2. Compression isotherm of 1)DPPG subphase PBS 2)DPPG subphase PalmE1(53-66)

\subsection{DSC}

The thermotropic fase behaviour of zwitterionic DPPC vesicles was studied by DSC, in the absence and in the presence of increasing concentrations of PalmE1(53-66) peptide derivative.

PalmE1(53-66) showed a slight decrease of the phase transition midpoint of the DPPC and a peak reduction (Fig.3). The onset temperature of the heating thermograms showed a near ideal behaviour, decreasing as the PalmE1(53-66) concentration increased (Fig 4.). The system evolved from a gel phase to a liquid-crystalline phase through a coexistence region which became wider as more peptide was added to the system. However, the completion or endset temperature behaved differently, not decreasing when the peptides concentration was increased, thus indicating that fluid immiscibility occurs.

Thus, having obtained a greater interaction of PalmE1(53-66) with DPPG, in future work its interaction with lipids of different charge will be studied in order to get more insight on the main forces involved in the interaction.

\section{ACKNOWLEDGEMENTS}

Grants BQU2000-0793-C02-01 and BQU2000-0793-C02-02 from the Ministerio de Ciencia y Tecnología (Spain) funded this work 


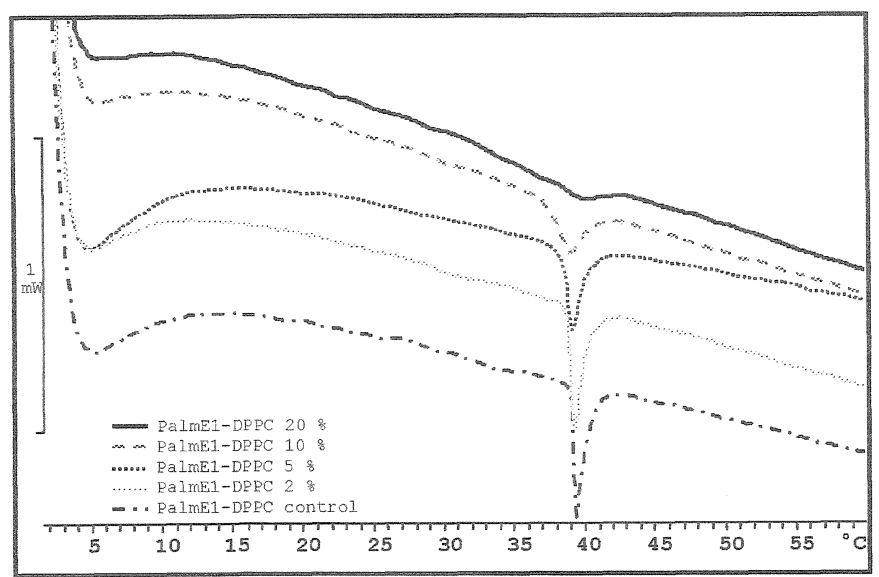

Figure 3. DPPC thermogram with PalmE1(53-66)

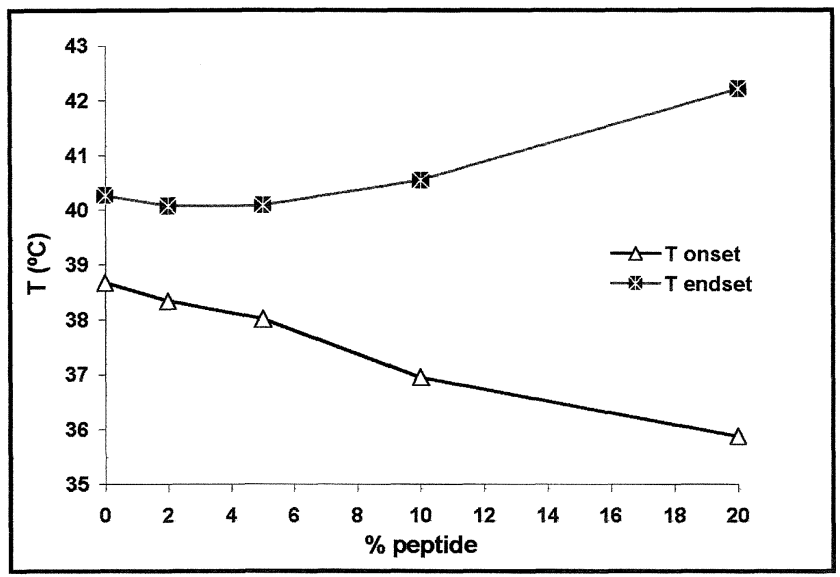

Figure 4. Temperatures endset and onset of DSC

1- T.P.Hopp, K.R. Woods, Proc. Natl. Acad. Sci. U.S.A., 78, 3824-3828 (1981).

2- G.W. Welling, W.J. Weijer, R. Van der Zee, S. Welling-Wester, FEBS Lett. 188, 215-218 (1985).

3- M.García, M. Pujol, F.Reig, M.A. Alsina, I. Haro, Analyst 121, 1583-1588 (1996).

4- M.J.Gómara, V. Girona, G. Ercilla, F. Reig, M.A. Alsina, I. Haro, Biopolymers 58(2) 117-28 (2001).

5- P. Sospedra, M. Espina, M.A. Alsina, I. Haro, C. Mestres, J Colloid and Interface Sci.244;87 (2001).

6- S. Taneva, D.R. Voelker, K.M. Keough, Biochemistry, 36(26), 173-9(1997). 